\title{
INFLUÊNCIAS DOS NÍVEIS DE RUGOSIDADE NOS RESULTADOS DE ANÁLISE QUÍMICA POR ESPECTROMETRIA DE EMISSÃO ÓPTICA
}

\author{
ARTIGO ORIGINAL \\ PEREIRA, Sérgio Rodrigo Araújo ${ }^{1}$ \\ BRAGA, Eduardo de Magalhães ${ }^{2}$
}

PEREIRA, Sérgio Rodrigo Araújo. BRAGA, Eduardo de Magalhães. Influências dos níveis de rugosidade nos resultados de análise química por espectrometria de emissão óptica. Revista Científica Multidisciplinar Núcleo do Conhecimento. Ano 05, Ed. 03, Vol. 09, pp. 102-121. Março de 2020. ISSN: 2448-0959, Link de acesso: https://www.nucleodoconhecimento.com.br/engenharia-mecanica/niveis-derugosidade

\section{RESUMO}

O presente estudo pretende averiguar as influências dos níveis de rugosidade, após análise química por espectrometria de emissão óptica, no intuito de verificar em qual etapa de lixamento a composição química do trilho de aço baixa liga (THH 370-JISE E1120), mais se aproximou do padrão original de fábrica. Mediu- se a rugosidade da superfície da amostra em vários níveis, (pós corte, \#60, \#120, \#220 e polido) e posteriormente, foi realizada a análise química por espectrometria de emissão óptica. Os resultados mostraram que a etapa de lixamento com mesh $120 \#$, apresentou os menores erros relativos de composição química e um dos parâmetros de rugosidade ( $\mathrm{Ra}, \mathrm{Rq}, \mathrm{Rz}$ ) mais homogêneos dentre as demais fases analisadas. A composição

\footnotetext{
${ }^{1}$ Acadêmico de Engenharia Mecânica (UFPA).

2 Doutorado em Engenharia Mecânica. Mestrado em Engenharia Mecânica. Graduação em Engenharia Mecânica.
} 
química do corpo de prova, após sucessivas etapas de lixamento e queimas por espectrômetro, oscilou de maneira distinta por elemento químico analisado.

Palavras-Chave: Rugosidade, espectrometria de emissão óptica, lixamento, aço de baixa liga, Parâmetros de Rugosidade.

\section{INTRODUÇÃO}

Durante o desenvolvimento de um projeto de engenharia, as propriedades dos materiais devem ser consideradas para determinar os esforços e as solicitações envolvidas. Como exemplo, no projeto de um eixo automotivo são verificadas todas as propriedades do aço de construção mecânica utilizado, bem como dos componentes que o acoplam. Com isso, torna-se fundamental que durante o processo de produção, sejam utilizados materiais que atendam as especificações determinadas em projeto. Para esse devido controle, procedimentos de ensaios mecânicos, físicos e químicos normatizados são realizados para o controle de qualidade ou verificação dos materiais (PAHL, 2005; ASHBY, 2011).

Nos aços a composição química possui elevada influência em suas propriedades físicas, mecânicas e químicas. Estas ligas possuem elementos base como o ferro (Fe) e carbono (C) (até 2,11\%), além de outros elementos químicos que promovem diferentes propriedades aos aços. Como por exemplo, a adição de cromo $(\mathrm{Cr})$ na liga aumenta à resistência mecânica, o endurecimento, a resistência ao desgaste abrasivo e à corrosão. Já a adição de tungstênio $(\mathrm{W})$ aumenta a dureza e reduz a condutividade térmica da liga de aço. Como no exemplo descrito, ambos os elementos químicos modificam as propriedades dos aços, ou seja, a ausência dos elementos essenciais ou a presença em demasia podem afetar as propriedades requerida na projeção do produto ou componente (CHIAVERINI, 2008).

Para determinar a composição química dos aços, um dos métodos utilizados é a espectrometria de emissão óptica. O princípio de funcionamento desse método/equipamento é baseado na medição das três grandezas físicas básicas da luz 
ou da onda eletromagnética: intensidade (ou amplitude), frequência e polarização (ângulo de vibração) (SERWAY, 2011).

\section{PROCEDIMENTO EXPERIMENTAL}

Com a finalidade de obter os resultados propostos neste estudo, foram realizados procedimentos experimentais. Os ensaios foram feitos no Laboratório de Caracterização de Materiais Metálicos (LCAM) da UFPA, os quais serão descritos detalhadamente os procedimentos, materiais e equipamentos utilizados.

Os trilhos usados para análise química são do modelo THH 370 (JISE 1120) de manufatura japonesa, cujo processo de fabricação é por meio da redução do minério de ferro em altos fornos para a produção do ferro gusa, posteriormente o material passa pela aciaria, laminação, tratamento térmico e alinhamento. A tabela 1 ilustra a composição química do trilho THH 370.

Tabela 1 - Composição química do trilho THH 370 (JISE 1120)

\begin{tabular}{|c|c|c|c|c|c|c|}
\hline C & Si & $\mathrm{Mn}$ & P & S & $\mathrm{Cr}$ & Outros \\
\hline 0,79 & 0,17 & 0,99 & $0,030 \leq$ & $0,020 \leq$ & 0,16 & $\begin{array}{l}\text { V:0,03 } \\
\text { máx }\end{array}$ \\
\hline
\end{tabular}

Fonte: (JFE, 2014).

$\mathrm{Na}$ tabela 1 identificam-se apenas os seis principais elementos constituintes da composição química do trilho $\mathrm{THH} 370$, fornecida pelo fabricante, os quais serão base na análise proposta.

Para atingir diferentes níveis de rugosidade, o corpo de prova submeteu-se a um processo de lixamento com diferentes granulometrias de lixas, conforme ilustrado na tabela 2. 
Tabela 2 - Identificação das diferentes preparações da superfície utilizadas no experimento.

\begin{tabular}{|l|c|c|c|c|c|}
\hline Identificação & 1 & 2 & 3 & 4 & 5 \\
\hline Granulometria & $\begin{array}{l}\text { Pós } \\
\text { corte }\end{array}$ & $\# 60$ & $\# 120$ & $\# 220$ & Polido \\
\hline
\end{tabular}

Fonte: (Autor).

Após o processo de lixamento com politriz metalográfica (modelo Fortec II), o corpo de prova foi submetido à limpeza com álcool isopropílico. A limpeza foi realizada para impedir a presença de contaminantes na análise da composição química. Posteriormente, avaliou-se a rugosidade do corpo de prova.

Para a medição da rugosidade, utilizou-se um rugosímetro portátil Mitutoyo, modelo Surft Sj 210, ajustado para medir 5 comprimentos de amostragem ( $\lambda s$ ) de 2,5 $\mu \mathrm{m}$ gerando um comprimento de avaliação (cut off) de 0,8 mm. As medições foram realizadas em 2 posições distintas, cumprindo à direção transversal (90) dos sulcos do corpo de prova, sendo considerada a média dos valores obtidos para as análises dos resultados. Além do perfil, a avaliação da rugosidade do perfil do corpo de prova, fora realizada nos parâmetros Ra, Rq e Rz (WHITEHOUSE, 2003). As figuras 1 e 2 ilustram o corpo de prova e o aparelho rugosímetro respectivamente.

Figura 1 - Corpo de prova analisado

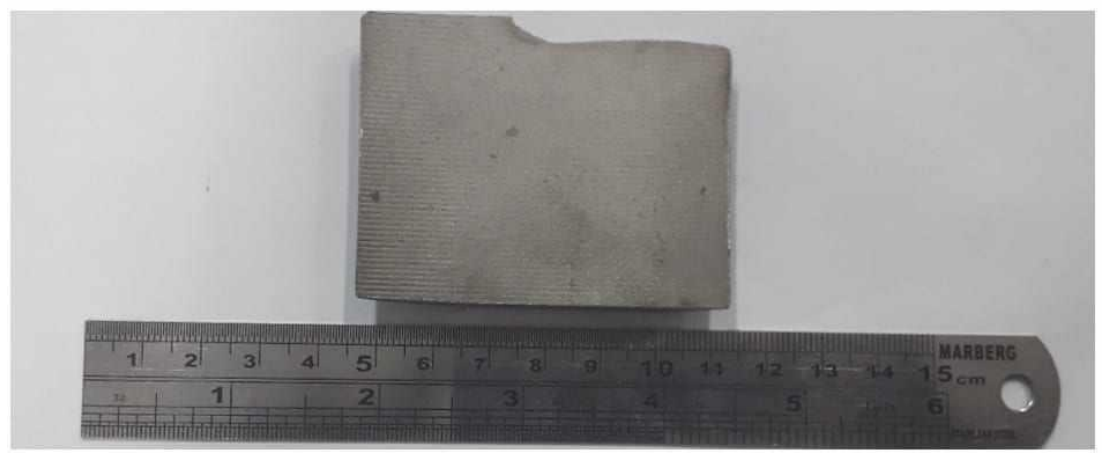

Fonte: (Autor).

Disponível em: https://www.nucleodoconhecimento.com.br/engenharia-mecanica/niveis-derugosidade 
Figura 2 - Rugosímetro portátil Mitutoyo Modelo Surft SJ 210

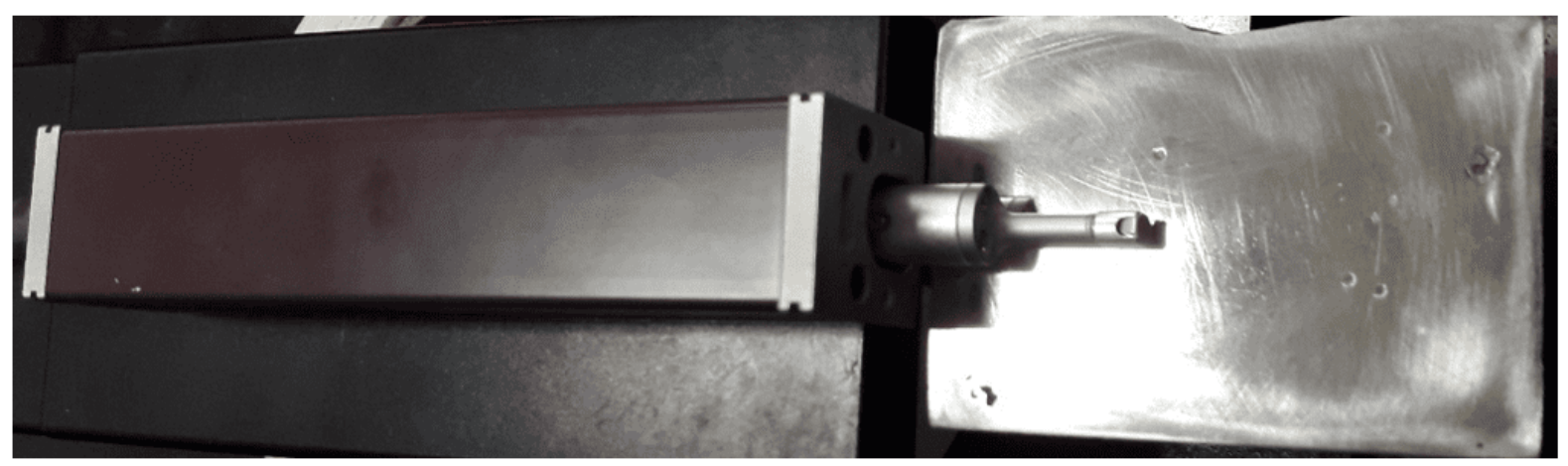

Fonte: (Autor).

Para o ensaio de composição química, foi usado o espectrômetro de emissão óptica, da fabricante Oxford, modelo Foundry Master Xpert. Salienta-se, que antes deste experimento, foram realizados os devidos procedimentos de acordo com as orientações do fabricante.

Inicialmente para evitar qualquer tipo de contaminação e imprecisão nos resultados, o instrumento foi purificado com gás argônio, comprimido sob pressão de 3,0 bar, 24 horas antes do início das análises, com auxílio de uma bomba a vácuo.

Figura 3 - Bomba a vácuo utilizada na purificação do espectrômetro

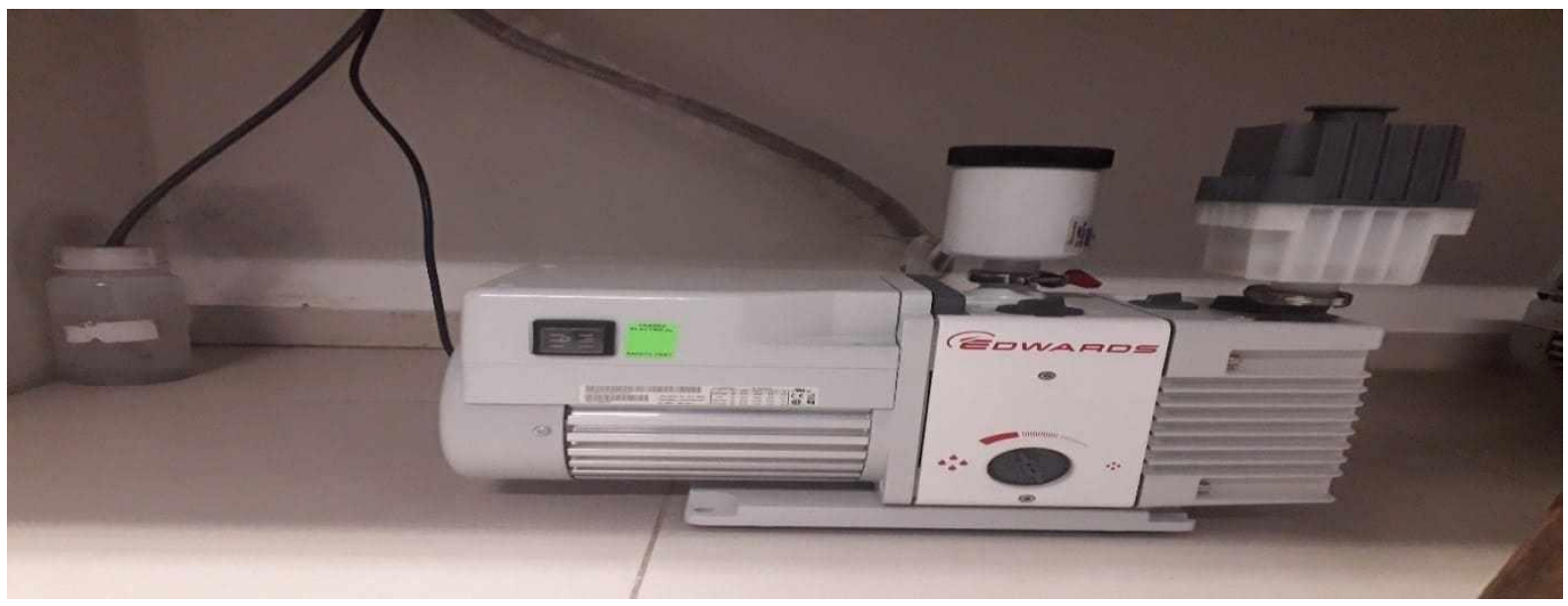

Fonte: (Autor).

Disponível em: https://www.nucleodoconhecimento.com.br/engenharia-mecanica/niveis-derugosidade 
O suporte de eletrodos, bem como o eletrodo usado para o centelhamento (neste caso, eletrodo de $\mathrm{Fe}$ ), foram limpos com uma escova. Logo após, o mesmo foi instalado no suporte, com o auxílio de uma chave Allen. O espaçamento entre a ponta do eletrodo e a superfície da amostra foi ajustado para 3,2 $\mathrm{mm}$ usando-se um espaçador, conforme orientações do manual do fabricante.

Para o tratamento dos dados, um computador foi ligado e o software Waslab, devidamente acionado. O programa analítico selecionado foi o Fe_100, ideal para análise de aços baixa liga. A amostra foi colocada no suporte de faísca com o lado a ser analisado para baixo.

Figura 4 - Espectrômetro de Emissão Óptica

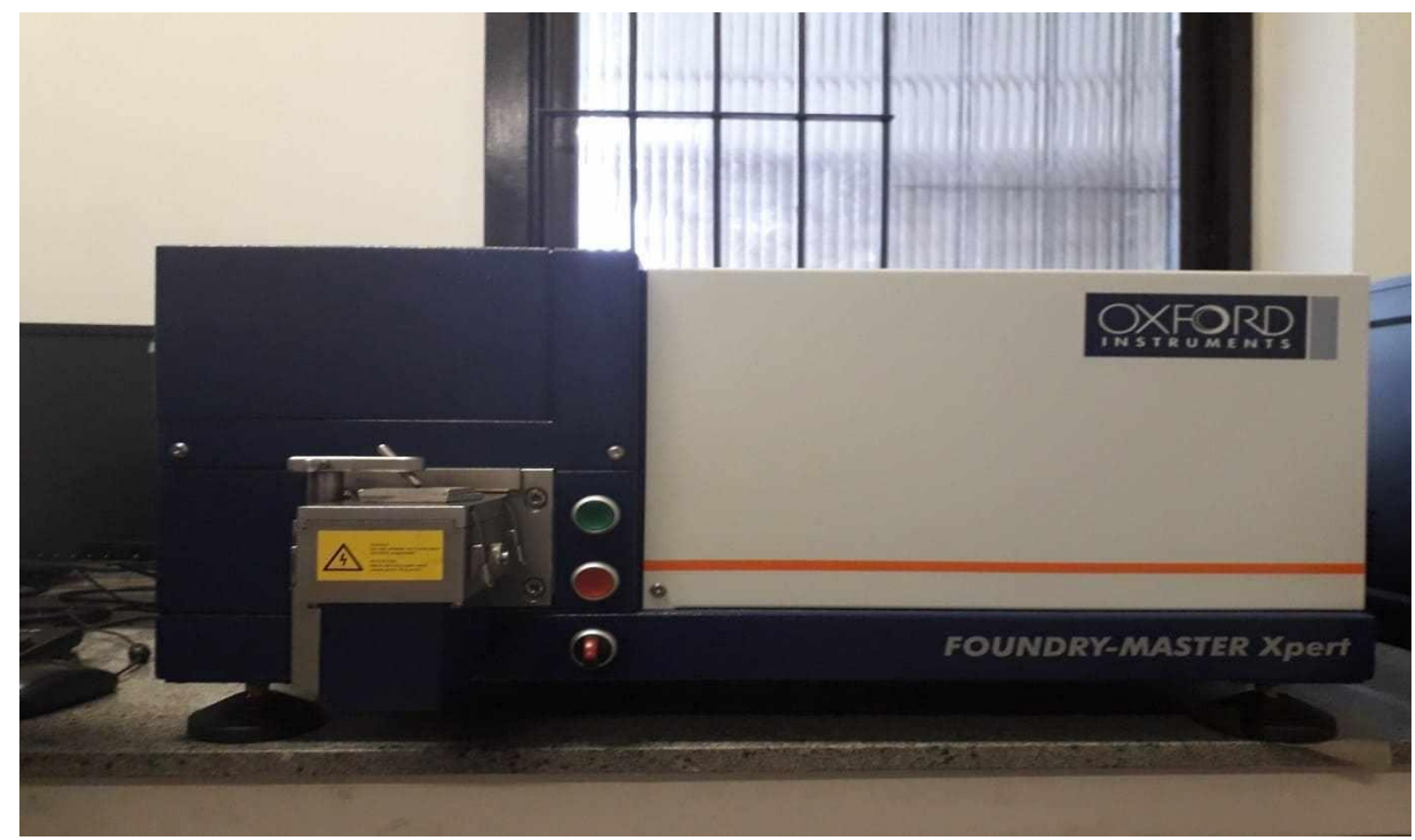

Fonte: (Autor).

Para cada preparação superficial (amostras 1 a 5), o número de queimas no espectrômetro foi repetido 10 vezes, possibilitando a comparação das amostras por análise de variância utilizando um intervalo de confiança de 99,7\%.

Disponível em: https://www.nucleodoconhecimento.com.br/engenharia-mecanica/niveis-derugosidade 
Por isso, cada ensaio consistia a realização da preparação da superfície do corpo de prova (padrão de calibração), dos procedimentos de lixamento, da medição de rugosidade e do ensaio de composição química. Para cada nova preparação, repetiuse os devidos procedimentos.

\section{RESULTADOS E DISCUSSÕES}

\subsection{ANÁLISE DA RUGOSIDADE}

\subsubsection{RUGOSIDADE - ETAPA 1}

De acordo com as superfícies geradas e com as etapas pré-definidas, estão dispostas as análises de rugosidade, sendo estas, medidas em dois sentidos: um longitudinal e outro no sentido transversal.

Abaixo estão listadas as médias das rugosidades encontradas no corpo de prova após os sucessivos lixamentos. $\mathrm{Na}$ tabela 3 , pode-se observar a média das rugosidades, nos sentidos longitudinal e transversal no corpo de prova após corte. É necessário enfatizar que o corte no corpo de prova foi feito por eletroerosão.

Tabela 3 - Medida de rugosidade longitudinal e transversal (pós-corte)

\begin{tabular}{|l|l|l|}
\hline Parâmetro & Longitudinal & Transversal \\
\hline $\mathrm{Ra}$ & $3,087 \mu \mathrm{m}$ & $4,070 \mu \mathrm{m}$ \\
\hline $\mathrm{Rq}$ & $3,702 \mu \mathrm{m}$ & $4,990 \mu \mathrm{m}$ \\
\hline $\mathrm{Rz}$ & $14,746 \mu \mathrm{m}$ & $21,584 \mu \mathrm{m}$ \\
\hline
\end{tabular}

Fonte: (Autor).

Como podemos observar, neste primeiro resultado, os valores Ra e Rq, encontram se bastante aproximados, enquanto há uma grande discrepância em relação ao valor obtido em Rz, tanto na longitudinal como na transversal, o que é normal em função de Rz ser um parâmetro que indica a altura dos pontos máximos e mínimos do perfil, 
sendo que, a diferença entre os valores na transversal está notoriamente mais acentuada. Isso se deve a forma de lixamento, neste caso, predominou o sentido transversal.

\subsubsection{RUGOSIDADE - ETAPA 2}

Posteriormente, a superfície do corpo de prova foi submetida a sua primeira etapa de lixamento com mesh \#60, obtendo os valores descritos na tabela 5 . Verifica-se uma brusca diminuição nos valores de rugosidade em relação à etapa anterior em ambos os sentidos da medição, todavia, há certa diferença favorável ao sentido transversal.

Tabela 4 - Medidas de Rugosidade Longitudinal e Transversal (\#60)

\begin{tabular}{|c|c|c|}
\hline Parâmetro & Longitudinal & Transversal \\
\hline $\mathrm{Ra}$ & $0,551 \mu \mathrm{m}$ & $1,291 \mu \mathrm{m}$ \\
\hline $\mathrm{Rq}$ & $0,756 \mu \mathrm{m}$ & $1,705 \mu \mathrm{m}$ \\
\hline $\mathrm{Rz}$ & $3,984 \mu \mathrm{m}$ & $9,709 \mu \mathrm{m}$ \\
\hline
\end{tabular}

Fonte: (Autor).

\subsubsection{RUGOSIDADE - ETAPA 3}

A próxima etapa de lixamento da superfície do corpo de prova, foi realizada com lixa mesh \#120, obedecendo os mesmos parâmetros do lixamento anterior. A medição de rugosidade dos sentidos longitudinal e transversal atingiu valores homogêneos. Obteve-se os seguintes valores apresentados na tabela 5.

Tabela 5 - Medidas de Rugosidade Longitudinal e Transversal (\#120)

\begin{tabular}{|l|l|l|}
\hline Parâmetro & Longitudinal & Transversal \\
\hline $\mathrm{Ra}$ & $0,807 \mu \mathrm{m}$ & $0,901 \mu \mathrm{m}$ \\
\hline $\mathrm{Rq}$ & $1,060 \mu \mathrm{m}$ & $1,184 \mu \mathrm{m}$ \\
\hline $\mathrm{Rz}$ & $5,687 \mu \mathrm{m}$ & $7,708 \mu \mathrm{m}$ \\
\hline
\end{tabular}


Fonte: (Autor).

\subsubsection{RUGOSIDADE - ETAPA 4}

Nesta fase, a superfície do corpo de prova foi lixada com lixa mesh \# 220, o qual observa-se, que os valores de rugosidade no sentido longitudinal se tornaram maiores que no sentido transversal pela primeira vez neste experimento, gerando os seguintes valores anexados na tabela 6.

Tabela 6 - Medidas de Rugosidade Longitudinal e Transversal (\#220)

\begin{tabular}{|l|l|l|}
\hline Parâmetro & Longitudinal & Transversal \\
\hline $\mathrm{Ra}$ & $0,248 \mu \mathrm{m}$ & $0,139 \mu \mathrm{m}$ \\
\hline $\mathrm{Rq}$ & $0,332 \mu \mathrm{m}$ & $0,180 \mu \mathrm{m}$ \\
\hline $\mathrm{Rz}$ & $1,619 \mu \mathrm{m}$ & $0,990 \mu \mathrm{m}$ \\
\hline
\end{tabular}

Fonte: (Autor).

\subsubsection{RUGOSIDADE - ETAPA 5}

Nesta última etapa, o corpo de prova foi submetido ao processo de polimento sendo lixado com diferentes tipos de lixa (\#40, \#60, \#120, \#220, \#600), e devidamente limpo para retirada de quaisquer resquícios metálicos. Obteve-se resultados de rugosidade, homogêneos em ambos os sentidos, sendo que, no sentido longitudinal os valores prevaleceram discretamente maiores. As medidas estão em apenso na tabela 7.

Tabela 7 - Medidas de Rugosidade Longitudinal e Transversal (Sup. Polida).

\begin{tabular}{|l|l|l|}
\hline Parâmetro & Longitudinal & Transversal \\
\hline $\mathrm{Ra}$ & $0,036 \mu \mathrm{m}$ & $0,031 \mu \mathrm{m}$ \\
\hline $\mathrm{Rq}$ & $0,049 \mu \mathrm{m}$ & $0,040 \mu \mathrm{m}$ \\
\hline $\mathrm{Rz}$ & $0,337 \mu \mathrm{m}$ & $0,261 \mu \mathrm{m}$ \\
\hline
\end{tabular}

Fonte: (Autor). 


\subsection{ANÁLISE QUIMICA POR (EEO)}

Posterior à fase de lixamento e medição de rugosidade, foi realizada à análise química por espectrometria de emissão óptica do corpo de prova. Para cada granulometria de lixa aplicada a superfície da peça de trabalho, mediu-se sua composição química, e com os dados obtidos, foi realizado análise de erro relativo para verificar qual nível de rugosidade mais se aproximou da composição química padrão do trilho em questão. A tabela 8, ilustra os valores obtidos, em todas as etapas de lixamento e seu desvio padrão absoluto, respectivamente.

Tabela 8 - Composição química da superfície do corpo de prova e desvio padrão.

\begin{tabular}{|c|c|c|c|c|c|c|c|}
\hline Mesh\# & C & $\mathrm{Si}$ & $\mathrm{Mn}$ & $P$ & & $S$ & $\mathrm{Cr}$ \\
\hline \multirow[t]{2}{*}{ Polido } & 0,858 & 0,250 & 1,19 & 0,0100 & \pm & 0,001 & $0,186 \pm$ \\
\hline & 0,015 & 0,0108 & $\pm \quad 0,009$ & 0,0008 & & 0,0011 & 0,0020 \\
\hline \multirow[t]{2}{*}{220} & 0,841 & $0,260 \pm$ & 1,17 & 0,0105 & \pm & 0,001 & $0,186 \pm$ \\
\hline & 0,017 & 0,0115 & 0,015 & 0,0002 & & 0,0010 & 0,0027 \\
\hline \multirow[t]{2}{*}{120} & 0,821 & $0,243 \pm$ & 1,20 & 0,0131 & \pm & 0,002 & $0,190 \pm$ \\
\hline & 0,013 & 0,0076 & 0,017 & 0,0001 & & 0,0012 & 0,0026 \\
\hline \multirow[t]{2}{*}{60} & 0,841 & $0,255 \pm$ & 1,19 & 0,0117 & \pm & 0,001 & $0,187 \pm$ \\
\hline & 0,024 & 0,0114 & 0,012 & 0,0001 & & 0,0013 & 0,0017 \\
\hline Pós & 0,916 & 0,247 & 1,20 & 0,0106 & \pm & 0,001 & $0,190 \pm$ \\
\hline corte & 0,048 & $\pm 0,0130$ & 0,001 & 0,0001 & & 0,0000 & 0,0022 \\
\hline
\end{tabular}

Fonte: (Autor).

\subsection{ERRO RELATIVO DOS ELEMENTOS QUÍMICOS}

De acordo com os dados descritos, o S teve a maior taxa de erro relativo, indicando que os seus valores estão relativamente menos próximos ao valor da norma, e, por sua vez, o $\mathrm{Cr}$ alcançou taxas de erro entre 0,1 e 1,5\%. A tabela 9 descreve os valores percentuais de erro relativo, conforme podemos observar. 
Tabela 9 - Erro relativo por elemento químico em (\%).

\begin{tabular}{|l|l|l|l|l|l|l|}
\hline & $\mathrm{C}$ & $\mathrm{Si}$ & $\mathrm{Mn}$ & $\mathrm{P}$ & $\mathrm{S}$ & $\mathrm{Cr}$ \\
\hline Pós corte & 16 & 45 & 22 & 64 & 95 & 0,1 \\
\hline \#60 & 6,4 & 53 & 20 & 61 & 93,5 & 1,5 \\
\hline \#120 & 3,9 & 41 & 21 & 56 & 90 & 0,1 \\
\hline \# 220 & 6,4 & 53 & 18 & 50 & 95 & 0,4 \\
\hline Polido & 47 & 47 & 20 & 66 & 94,5 & 0,4 \\
\hline
\end{tabular}

Fonte: (Autor).

Nota- se, que nas etapas de lixamento \#120 e \#220, há maior homogeneidade nos resultados, ilustrando as duas menores séries de taxas de erro relativo em relação aos demais estágios de lixamento.

\section{CONCLUSÃO}

Com base nos dados obtidos, observou - se que os valores encontrados nos parâmetros $\mathrm{Ra}, \mathrm{Rq}$ e $\mathrm{Rz}$ no mesh 120\#, tiveram um dos comportamentos mais homogêneos dentre os demais, e com menor taxa de erro relativo de composição química do trilho de aço baixa liga. O parâmetro $\mathrm{Rz}$, foi o que proporcionou maiores valores, o que já era esperado, em função do mesmo ser a média entre os pontos mínimos e máximos de rugosidade da linha média, enquanto os parâmetros $R a \mathrm{e} R q$, tiveram comportamento homogêneo, com tímida oscilação no decorrer das etapas. A partir do lixamento com mesh $220 \#$ os valores de rugosidade no sentido longitudinal se tornaram maiores que no sentido transversal, todavia, todos os parâmetros após esta etapa apresentaram valores de rugosidade bastante aproximados.

O lixamento com mesh 120\# apresentou menor erro relativo em 4/6 dos elementos analisados, sendo estes: C, Si, S e Cr. É importante ressaltar que nos elementos químicos $\mathrm{P}$ e $\mathrm{Mn}$, seu erro relativo foi o segundo menor. 
Nas etapas onde os parâmetros de rugosidade apresentaram maior homogeneidade, tanto no sentido transversal como no sentido longitudinal, as taxas de erro relativo foram as menores.

Portanto, o lixamento com mesh 120\#, mostrou- se uma solução rápida e barata de tratamento superficial, haja vista, baseado nos dados adquiridos, que sua ação em aço de baixa liga, se aproximou, em termos de composição química, aos níveis estabelecidos pelo fabricante do trilho THH 370 (JISE E1120).

\section{REFERÊNCIAS}

ASHBY, Michael F. Materials selection in mechanical design. 4th ed. Amsterdam: Elsevier, 2011. 646 p. 17

CHIAVERINI, Vicente. Aços e ferros fundidos: características gerais, tratamentos térmicos, principais tipos. 7. ed. ampl. e rev. São Paulo: Associação Brasileira de Metalurgia e Materiais, 2008. 599 p.

JFE-STEELCO, 2014. http://www.jfe-steel.co.jp/en/release/2014.html. Acessado em: 17/02/2019.

PAHL, Gerhard. Projeto na engenharia: fundamentos do desenvolvimento eficaz de produtos, métodos e aplicações. São Paulo: Edgard Blücher, 2005. 412 p.

SERWAY, Raymond A; JEWETT Jr, John W. Física para cientistas e engenheiros: eletricidade e magnetismo. São Paulo: Cengage Learning, 2011.

WHITEHOUSE, D. J. Handbook of surface and nanometrology. 1 ed. ed. Bristol: IPP Publisher, 2003. 1128 p. 


\section{ANEXOS}

\section{ANEXO I - Certificado de Calibração do Espectrômetro}

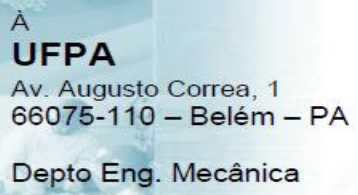

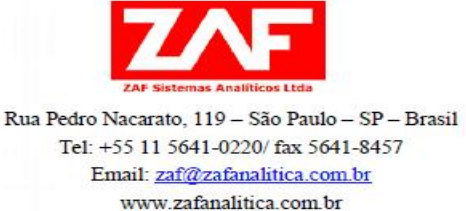

wrow zafanaticacom

\section{CERTIFICADO DE CALIBRAÇÃO}

No: 52P0061/1802.1

Instrumento:

Marca:

Modelo:

Número de série:

Aplicação:

Tensão de trabalho:

Detector(es)

Data da calibração:

Validade da calibração
FRÉTRICA:

Chave de tensão do eletrodo:

Bomba de vácuo na câmara ótica

Aterramento do chassis:

Estrutura física de blindagem:

Sensor de trava de amostra:

ESPECIFICAÇÖES TÉCNICAS: PROCEDIMENTO Oxford Instruments - OESPMCLST

Vácuo $(<0.1000$ mBAR):

Qualidade do gás Argônio:

Pressão do gás Argônio (3 a 4 kgf)

Caminho ótico:

Espectrômetro de Emissão Óptica

Oxford Instruments - IAG.

Foundry Master Xpert

52P0061

Ligas de $\mathrm{Al}, \mathrm{Co}, \mathrm{Cu}, \mathrm{Fe}, \mathrm{Ni}$ e $\mathrm{Ti}$

220V

CCD (16x)

Fevereiro de 2018

12 meses, observado o procedimento de uso correto

Luz de identificação ativa

Funcionamento adequado

Corretamente instalado

Permanece inalterada

Funcionamento adequado

Estabilidade de resultados após $4 \mathrm{hrs}$

Analítico 5.0

$3.5 \mathrm{kgf}$

Lentes e janelas em condiçöes normais de uso

AMOSTRAS SUS (Set Up Samples)

Coeficientes de calibração (0.5 a 3.0):

Todos dentro da faixa

SUS utilizadas:

RE 12/148, RG 14/320, RH 18/41, RN 19/40, RH 34/3

CONCLUSÃO

O equipamento está em plena capacidade de funcionamento, dentro dos parâmetros exigidos pelo fabricante.

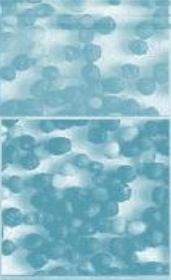

Disponível em: https://www.nucleodoconhecimento.com.br/engenharia-mecanica/niveis-de- 
ANEXO II - Certificado de Análise de Rugosidade (Pós corte - longitudinal)

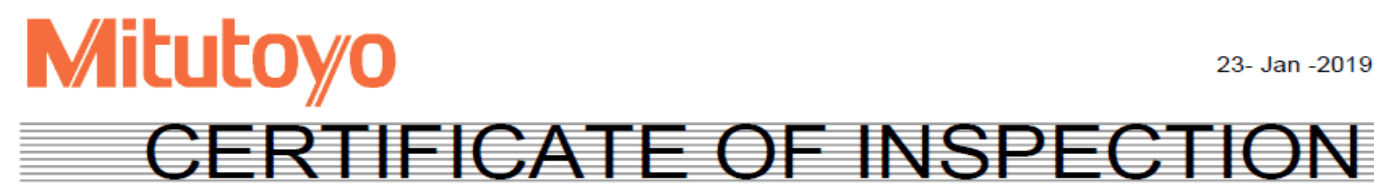

茞

Evaluation Profile

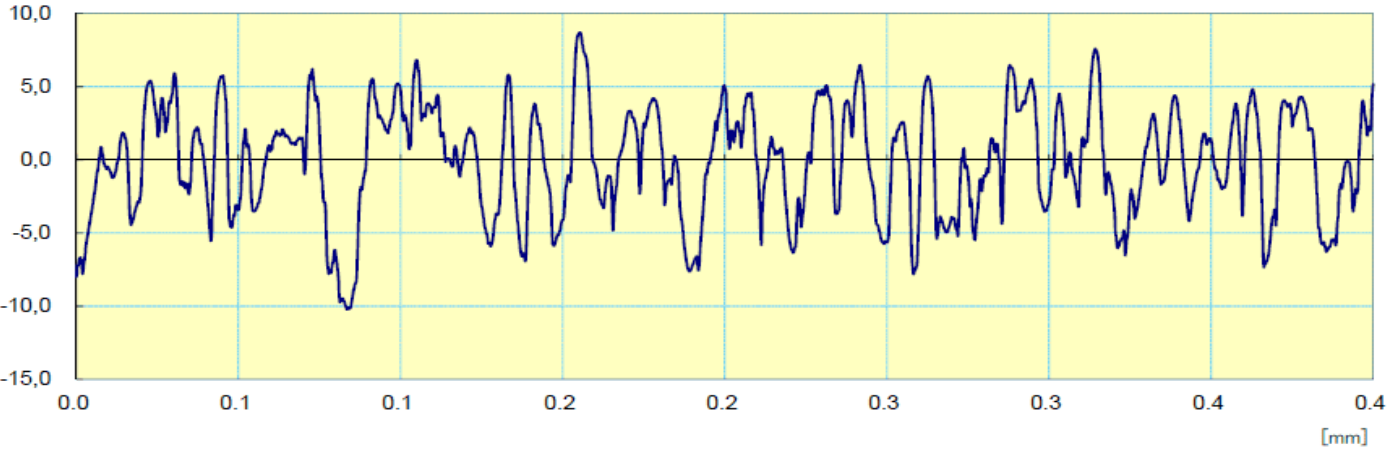

\begin{tabular}{cccc}
\hline Teste rugosidade & CP 01 & Operador & Tárcio \\
\hline Measuring Tool & SurfTest & Comment & Ver2.00 \\
\hline Standard & & N & 5 \\
\hline Profile & ISO 1997 & Cut-Off & GAUSS \\
\hline$\lambda s$ & $2.5 \mu \mathrm{m}$ & Filter & \\
\hline $\mathrm{Ra}$ & $3.087 \mu \mathrm{m}$ & & \\
\hline $\mathrm{Rq}$ & $3.702 \mu \mathrm{m}$ & & \\
\hline $\mathrm{Rz}$ & $14.746 \mu \mathrm{m}$ & \\
\hline & & \\
\hline & & \\
\hline
\end{tabular}

Copyright (C) 2013 Mitutoyo Corporation

RC: 47625

Disponível em: https://www.nucleodoconhecimento.com.br/engenharia-mecanica/niveis-derugosidade 


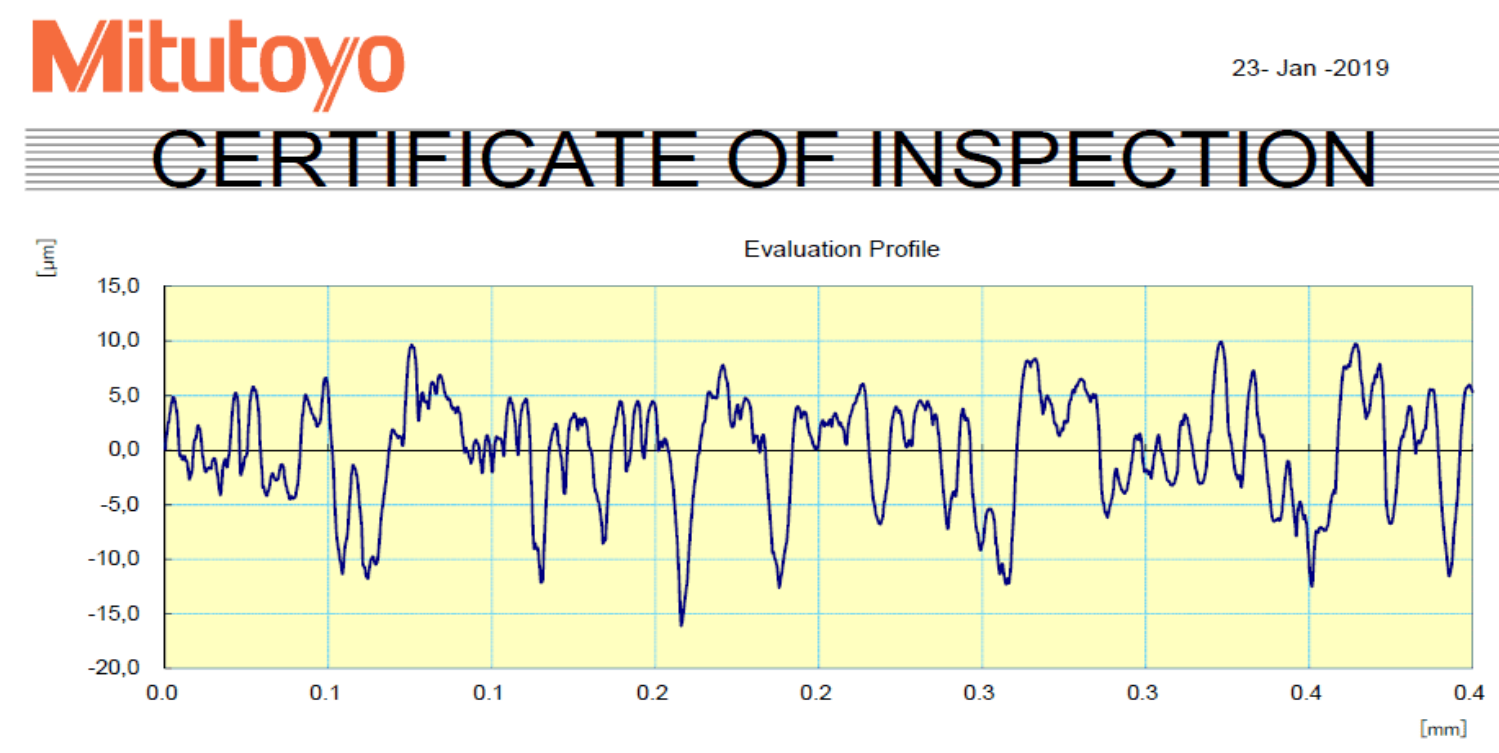

\begin{tabular}{cccc}
\hline Teste rugosidade & CP 01 & Operador & Tárcio \\
\hline Measuring Tool & Surfest & Comment & Ver2.00 \\
\hline Standard & & & \\
\hline Profile & SO 1997 & Cut-Off & GAUSS \\
\hline$\lambda s$ & $2.5 \mu \mathrm{m}$ & Filter & \\
\hline $\mathrm{Ra}$ & $4.070 \mu \mathrm{m}$ & & \\
\hline $\mathrm{Rq}$ & $4.990 \mu \mathrm{m}$ & & \\
\hline $\mathrm{Rz}$ & $21.584 \mu \mathrm{m}$ & & \\
\hline & & \\
\hline
\end{tabular}

Disponível em: https://www.nucleodoconhecimento.com.br/engenharia-mecanica/niveis-derugosidade 


\section{CERTIFICATE OF INSPECTION}

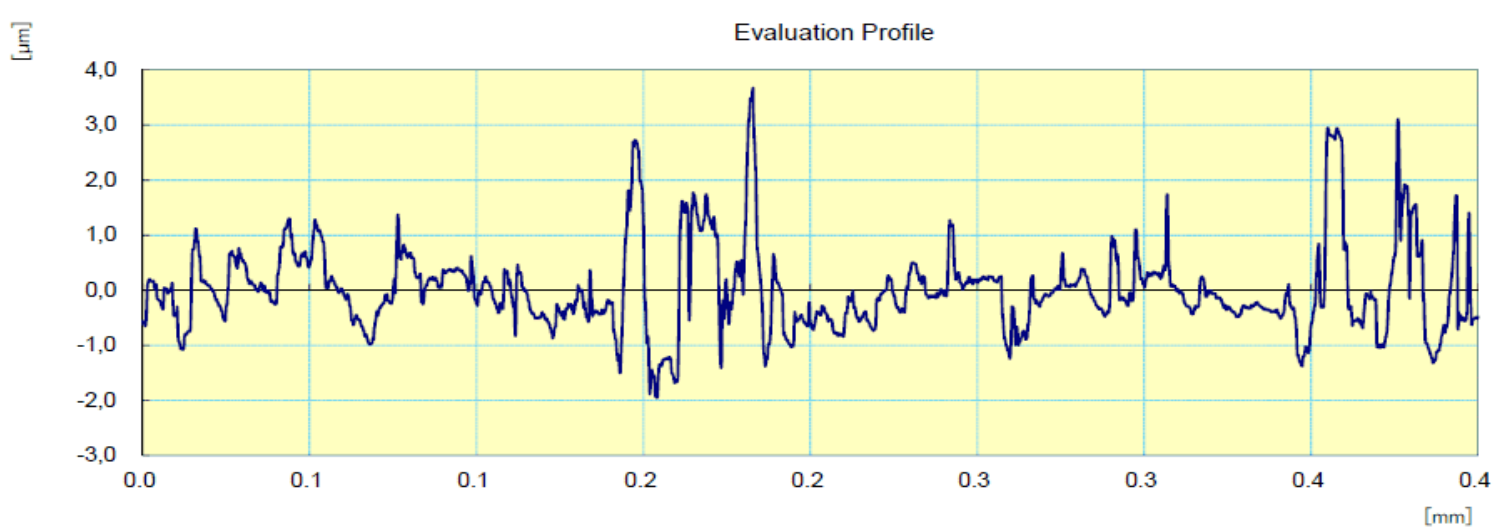

\begin{tabular}{cccc}
\hline Teste rugosidade & CP 01 & Operador & Tárcio \\
\hline Measuring Tool & Surfest & Comment & Ver2.00 \\
\hline Standard & & & 5 \\
\hline Profile & ISO 1997 & Cut-Off & GAUSS \\
\hline$\lambda s$ & $2.5 \mu \mathrm{m}$ & Filter & \\
\hline $\mathrm{Ra}$ & $0.551 \mu \mathrm{m}$ & & \\
\hline $\mathrm{Rq}$ & $0.756 \mu \mathrm{m}$ & & \\
\hline $\mathrm{Rz}$ & $3.984 \mu \mathrm{m}$ & & \\
\hline & & \\
\hline & & \\
\hline
\end{tabular}

Disponível em: https://www.nucleodoconhecimento.com.br/engenharia-mecanica/niveis-derugosidade 
ANEXO V - Certificado de Análise de Rugosidade (Mesh 60\# - transversal)

Evaluation Profile

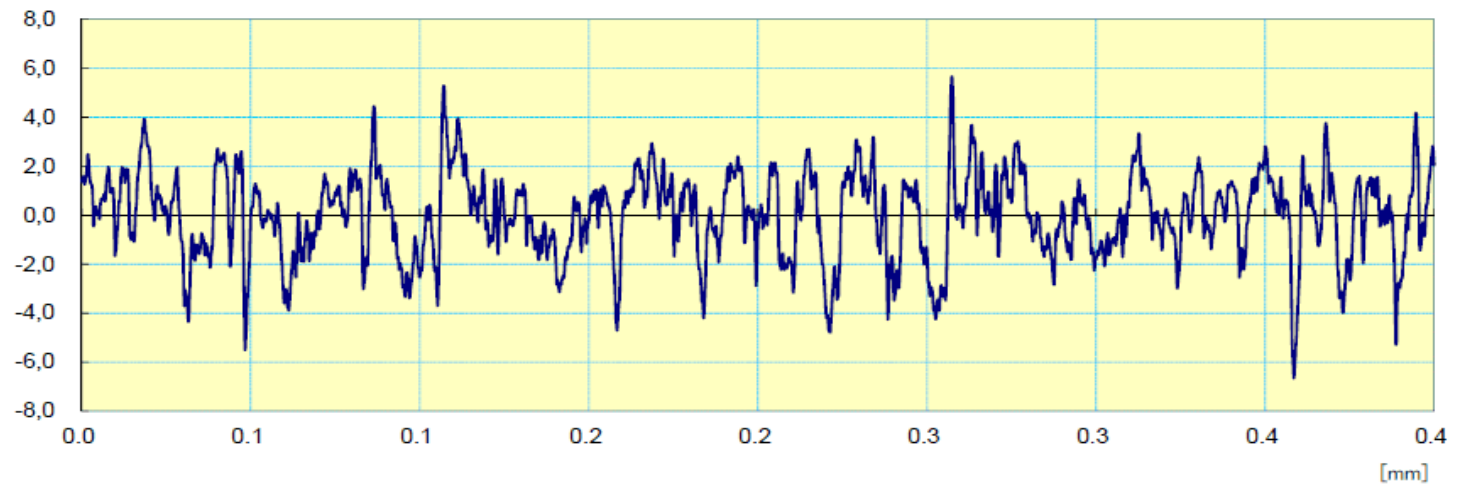

\begin{tabular}{|c|c|c|c|}
\hline Teste rugosidade & CP 01 & Operador & Tárcio \\
\hline Measuring Tool & SurfTest & Comment & Ver2.00 \\
\hline Standard & ISO 1997 & $\mathrm{~N}$ & 5 \\
\hline Profile & $\mathrm{R}$ & Cut-Off & $0.8 \mathrm{~mm}$ \\
\hline$\lambda s$ & $2.5 \mu \mathrm{m}$ & Filter & GAUSS \\
\hline $\mathrm{Ra}$ & $1.380 \mu \mathrm{m}$ & & \\
\hline $\mathrm{Rq}$ & $1.740 \mu \mathrm{m}$ & & \\
\hline $\mathrm{Rz}$ & $9.626 \mu \mathrm{m}$ & & \\
\hline & & & \\
\hline & & & \\
\hline & & & \\
\hline & & & \\
\hline & & & \\
\hline & & & \\
\hline & & & \\
\hline
\end{tabular}

Disponível em: https://www.nucleodoconhecimento.com.br/engenharia-mecanica/niveis-derugosidade 


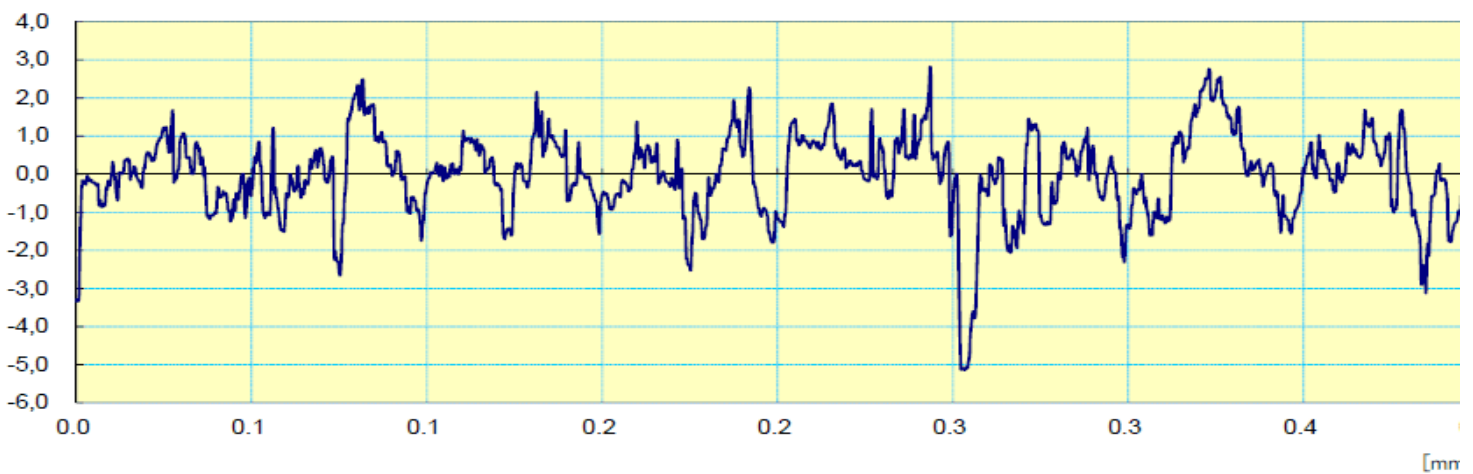

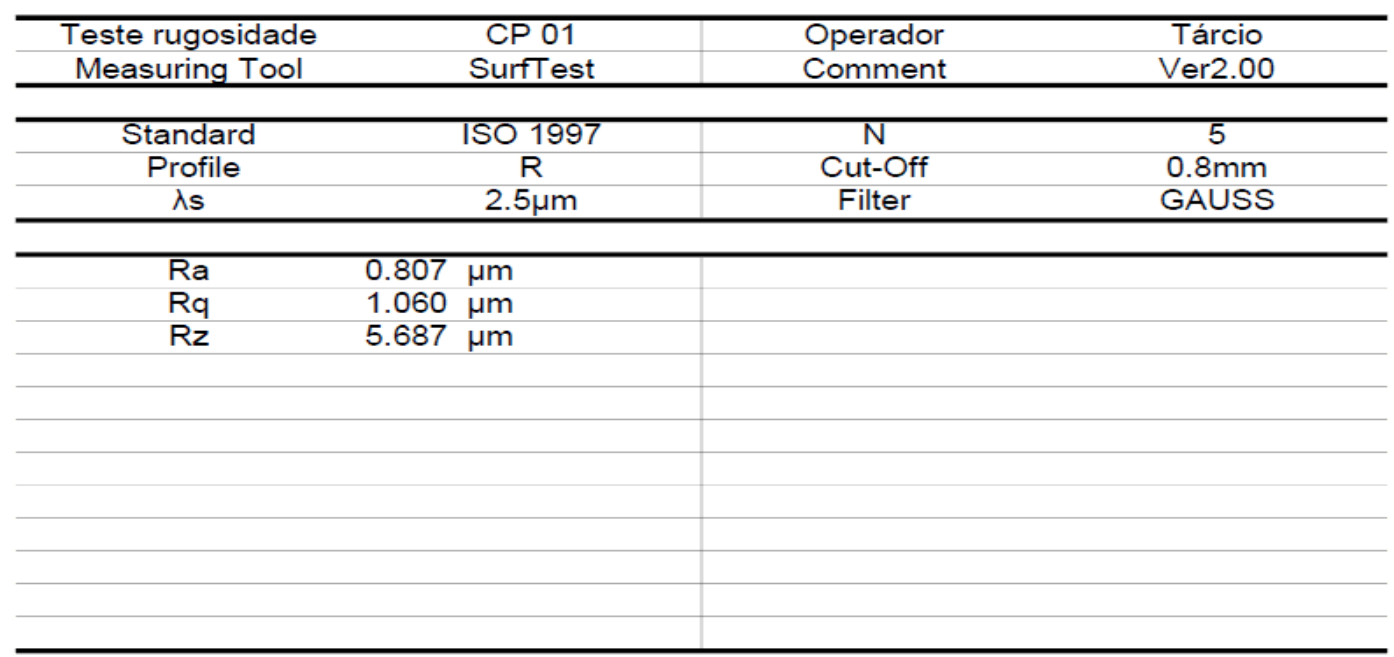

Disponível em: https://www.nucleodoconhecimento.com.br/engenharia-mecanica/niveis-derugosidade 


\section{CERTIFICATE OF INSPECTION}

写

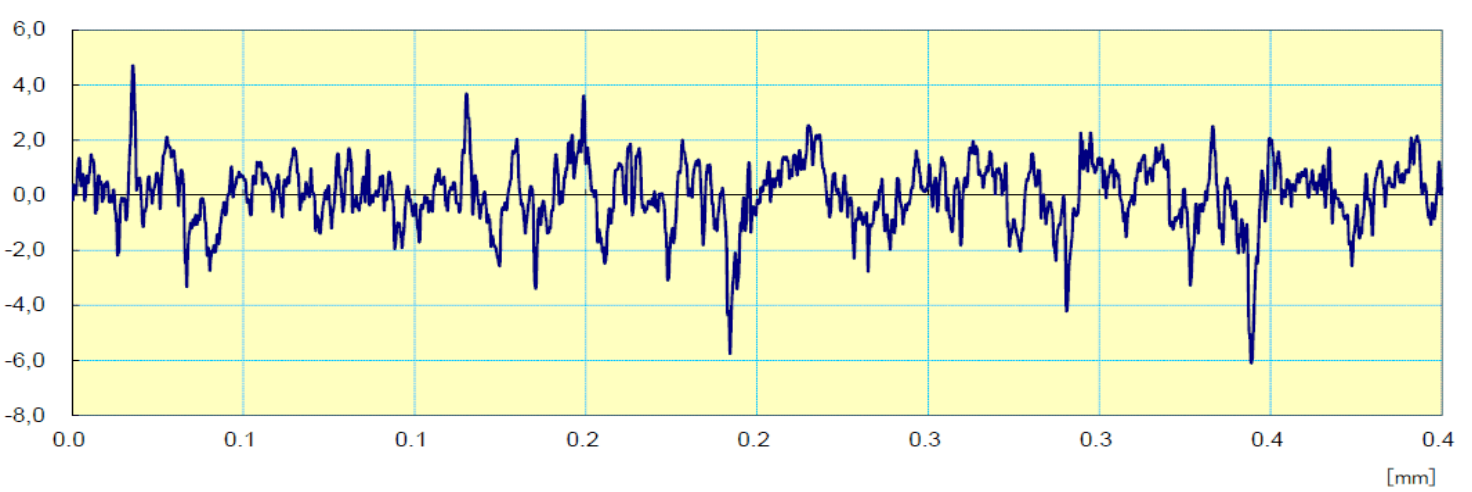

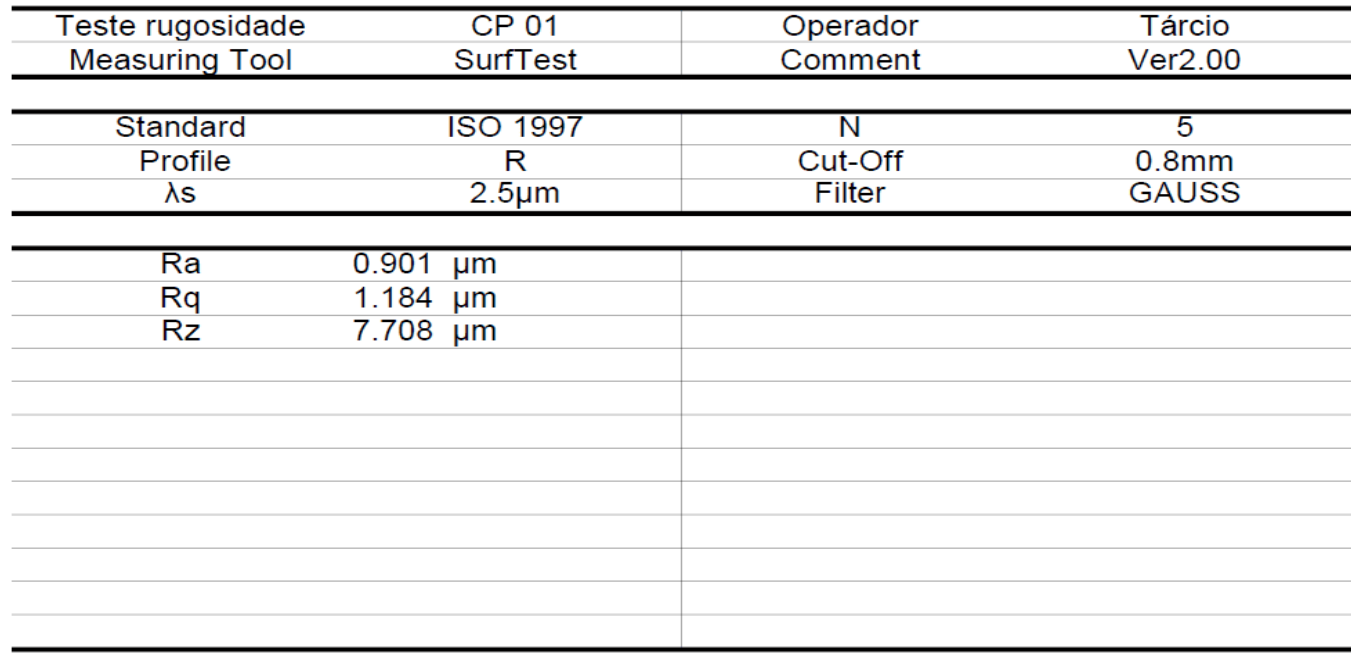

Disponível em: https://www.nucleodoconhecimento.com.br/engenharia-mecanica/niveis-derugosidade 
ANEXO VIII - Certificado de Análise de Rugosidade (Mesh 220\# -longitudinal)

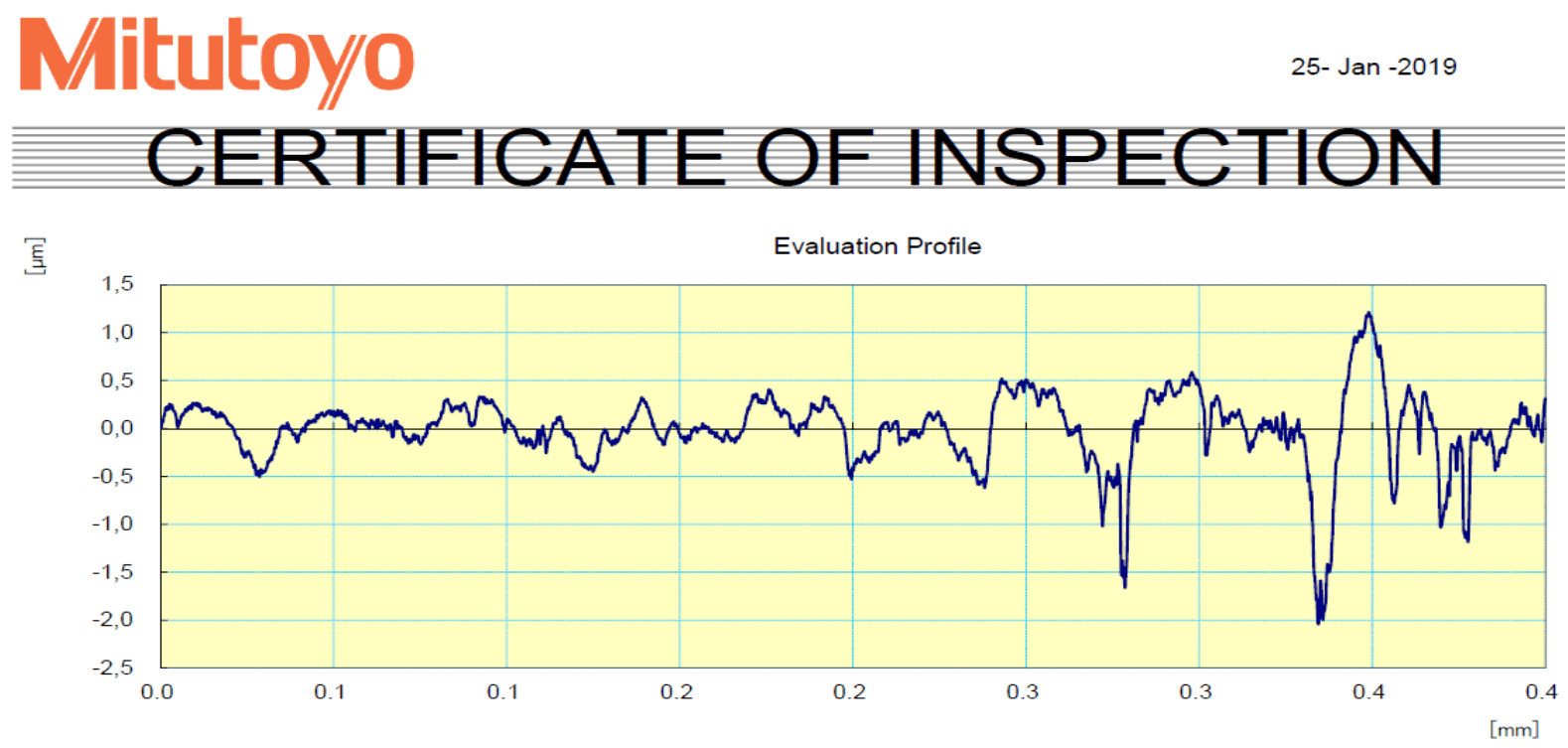

\begin{tabular}{cccc}
\hline Teste rugosidade & CP 01 & Operador & Tárcio \\
\hline Measuring Tool & SurfTest & Comment & Ver2.00 \\
\hline Standard & & & 5 \\
\hline Profile & ISO 1997 & Cut-Off & $0.8 \mathrm{~mm}$ \\
\hline$\lambda \mathrm{s}$ & $\mathrm{R}$ & Filter & GAUSS \\
\hline $\mathrm{Ra}$ & $2.5 \mu \mathrm{m}$ & & \\
\hline $\mathrm{Rq}$ & $0.248 \mu \mathrm{m}$ & & \\
\hline $\mathrm{Rz}$ & $0.332 \mu \mathrm{m}$ & & \\
\hline & $1.619 \mu \mathrm{m}$ & & \\
\hline & & & \\
\hline & & & \\
\hline & & & \\
\hline & & \\
\hline
\end{tabular}

Disponível em: https://www.nucleodoconhecimento.com.br/engenharia-mecanica/niveis-derugosidade 


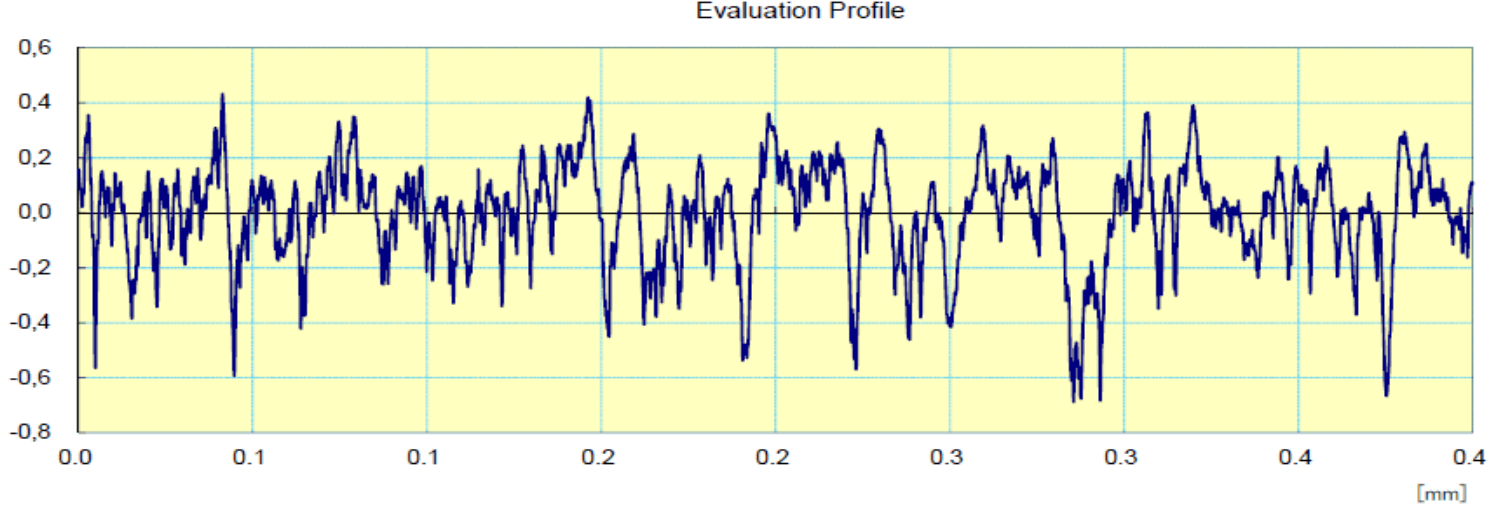

\begin{tabular}{cccc}
\hline Teste rugosidade & CP 01 & Operador & Tárcio \\
\hline Measuring Tool & Surfest & Comment & Ver2.00 \\
\hline Standard & & N & 5 \\
\hline Profile & SO 1997 & Cut-Off & GAUSS \\
\hline$\lambda s$ & $2.5 \mu \mathrm{m}$ & Filter & \\
\hline $\mathrm{Ra}$ & $0.139 \mu \mathrm{m}$ & & \\
\hline $\mathrm{Rq}$ & $0.180 \mu \mathrm{m}$ & & \\
\hline $\mathrm{Rz}$ & $0.990 \mu \mathrm{m}$ & \\
\hline & & \\
\hline & & \\
\hline & & \\
\hline & & \\
\hline
\end{tabular}

Disponível em: https://www.nucleodoconhecimento.com.br/engenharia-mecanica/niveis-derugosidade 
ANEXO X - Certificado de Análise de Rugosidade (polido- longitudinal)

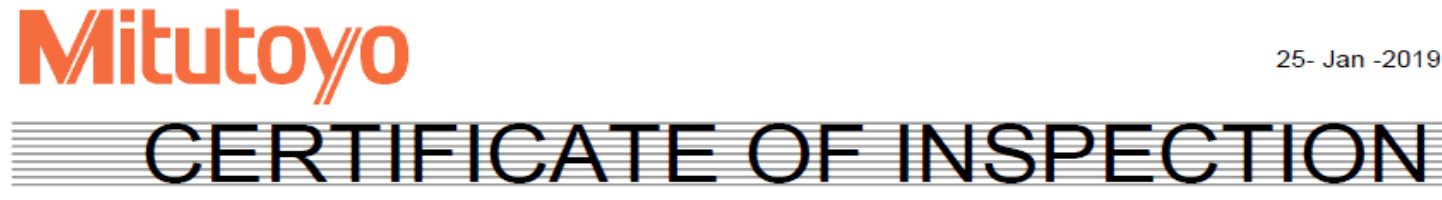

当

Evaluation Profile

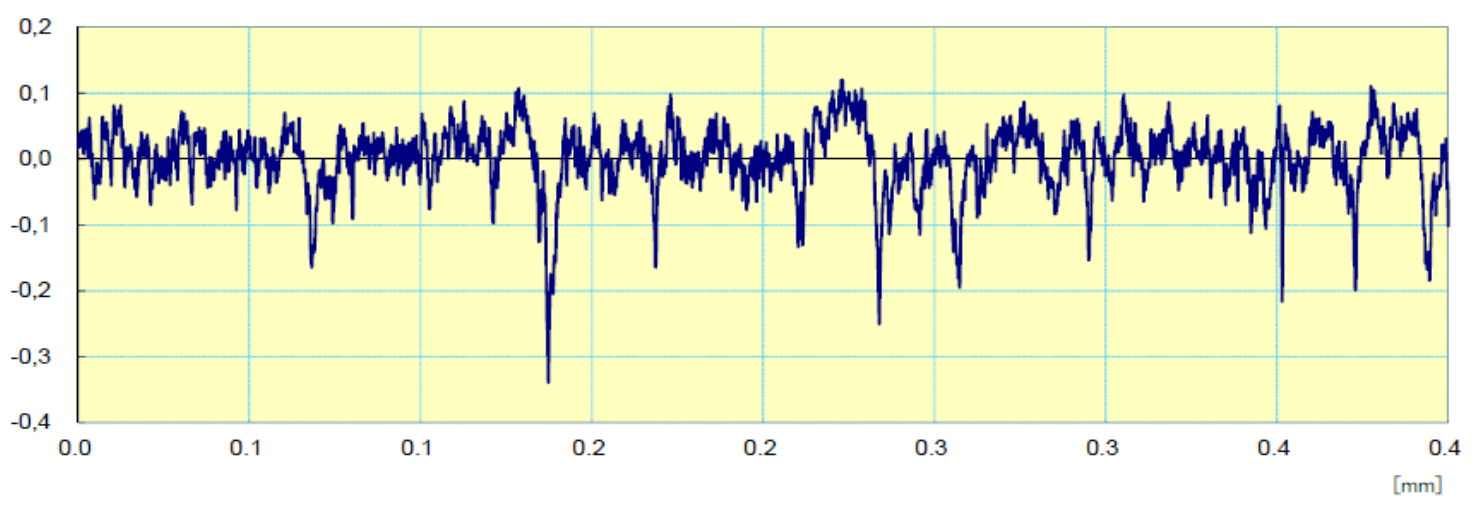

\begin{tabular}{cccc}
\hline Teste rugosidade & CP 01 & Operador & Tárcio \\
\hline Measuring Tool & SurfTest & Comment & Ver2.00 \\
\hline Standard & & & \\
\hline Profile & SO 1997 & Cut-Off & GAUSS \\
\hline$\lambda s$ & $2.5 \mu \mathrm{m}$ & Filter & \\
\hline $\mathrm{Ra}$ & $0.036 \mu \mathrm{m}$ & & \\
\hline $\mathrm{Rq}$ & $0.049 \mu \mathrm{m}$ & & \\
\hline $\mathrm{Rz}$ & $0.337 \mu \mathrm{m}$ & & \\
\hline & & \\
\hline & & \\
\hline
\end{tabular}

Copyright (C) 2013 Mitutoyo Corporation

RC: 47625

Disponível em: https://www.nucleodoconhecimento.com.br/engenharia-mecanica/niveis-derugosidade 
ANEXO XI - Certificado de Análise de Rugosidade (polido- transversal)

\section{Militutoy/0

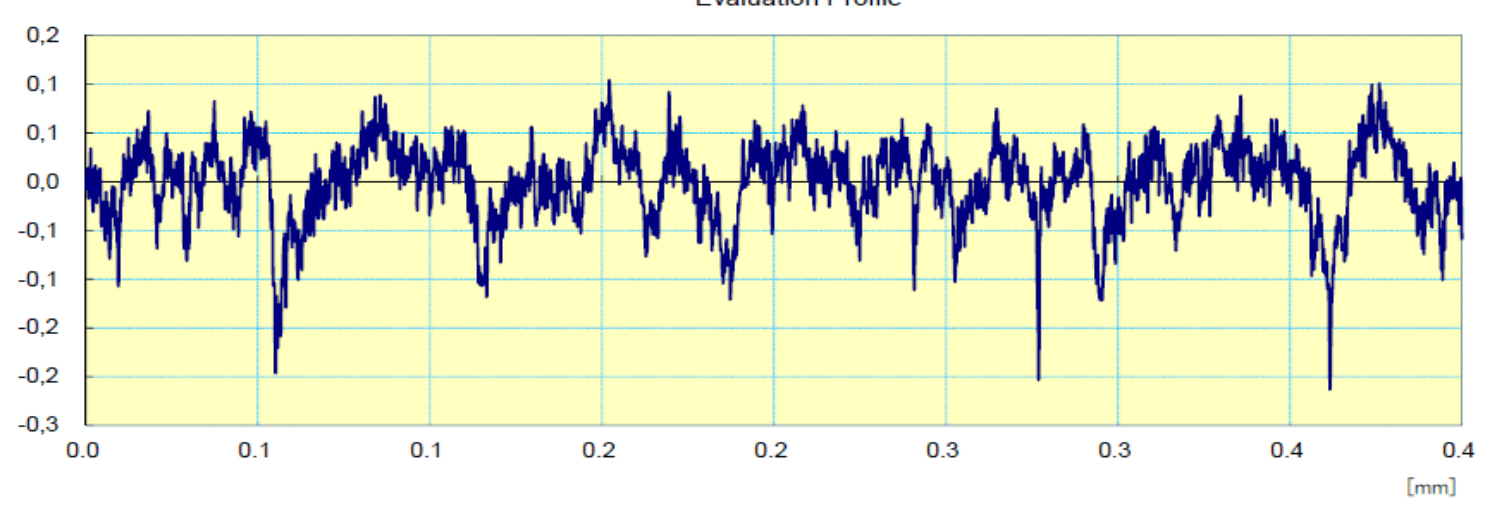

\begin{tabular}{cccc}
\hline Teste rugosidade & CP 01 & Operador & Tárcio \\
\hline Measuring Tool & Surfest & Comment & Ver2.00 \\
\hline Standard & & & \\
\hline Profile & SO 1997 & Cut-Off & GAUSS \\
\hline$\lambda s$ & $2.5 \mu \mathrm{m}$ & Filter & \\
\hline $\mathrm{Ra}$ & $0.031 \mu \mathrm{m}$ & & \\
\hline $\mathrm{Rq}$ & $0.040 \mu \mathrm{m}$ & & \\
\hline $\mathrm{Rz}$ & $0.261 \mu \mathrm{m}$ & & \\
\hline & & \\
\hline
\end{tabular}

Disponível em: https://www.nucleodoconhecimento.com.br/engenharia-mecanica/niveis-derugosidade 
Enviado: Outubro, 2019.

Aprovado: Março, 2020.

Disponível em: https://www.nucleodoconhecimento.com.br/engenharia-mecanica/niveis-derugosidade 